\title{
Associations between Interleukin-31 Gene Polymorphisms and Dilated Cardiomyopathy in a Chinese Population
}

\author{
Huizi Song, ${ }^{1,2}$ Ying Peng, ${ }^{1}$ Bin Zhou, ${ }^{3}$ Nan Chen, ${ }^{2}$ Xiaochuan Xie,,2 Qingyu Dou, ${ }^{1}$ \\ Yue Zhong, ${ }^{1,2}$ and Li Rao' \\ ${ }^{1}$ Department of Cardiology, West China Hospital of Sichuan University, Chengdu 610041, China \\ ${ }^{2}$ West China School of Medicine/West China Hospital of Sichuan University, Chengdu 610041, China \\ ${ }^{3}$ Laboratory of Molecular Translational Medicine, Key Laboratory of Obstetric \& Gynecology and Pediatric Diseases and Birth \\ Defects of Ministry of Education, West China Institute of Women's and Children's Health/West China Second University Hospital, \\ Sichuan University, Chengdu, Sichuan, China
}

Correspondence should be addressed to Li Rao; raoli8866@163.com

Received 22 January 2017; Revised 4 March 2017; Accepted 28 March 2017; Published 10 May 2017

Academic Editor: Agata M. Bielecka-Dabrowa

Copyright (C) 2017 Huizi Song et al. This is an open access article distributed under the Creative Commons Attribution License, which permits unrestricted use, distribution, and reproduction in any medium, provided the original work is properly cited.

To explore the role of Interkeulin-31 (IL-31) in dilated cardiomyopathy (DCM), in our study, two SNPs of IL-31, rs4758680 $(\mathrm{C} / \mathrm{A})$ and rs7977932 (C/G), were analyzed in $331 \mathrm{DCM}$ patients and 493 controls in a Chinese Han population. The frequencies of $\mathrm{C}$ allele and CC genotype of rs 4758680 were significantly increased in DCM patients $(P=0.005, P=0.001$, resp.). Compared to CC genotype of rs4758680, the A carriers (CA/AA genotypes) were the protect factors in DCM susceptibility while the frequencies of CA/AA genotypes were decreased in the dominant model for DCM group $(P<0.001$, $\mathrm{OR}=0.56,95 \% \mathrm{CI}=0.39-0.79)$. Moreover, IL-31 mRNA expression level of white blood cells was increased in DCM patients (0.072 (0.044-0.144) versus $0.036(0.020-0.052), P<0.001)$. In survival analysis of 159 DCM patients, Kaplan-Meier curve revealed the correlation between CC homozygote of rs4758680 and worse prognosis for DCM group $(P=0.005)$. Compared to CC genotype, the CA/AA genotypes were the independent factors in both univariate $(\mathrm{HR}=0.530,95 \% \mathrm{CI}=0.337-0.834$, $P=0.006)$ and multivariate analyses after age, gender, left ventricular end-diastolic diameter, and left ventricular ejection fraction adjusted $(\mathrm{HR}=0.548,95 \% \mathrm{CI}=0.345-0.869, P=0.011)$. Thus, we concluded that $I L-31$ gene polymorphisms were tightly associated with DCM susceptibility and contributed to worse prognosis in DCM patients.

\section{Introduction}

Dilated cardiomyopathy (DCM) as a primary myocardial disease is marked by dilation of the left ventricle as well as systolic dysfunction that is with progressive functional and structural changes $[1,2]$. It affects $-1 / 2500$ adults and more common in men than in women $[3,4]$. DCM is one of the pivotal causes of sudden cardiac death and congestive heart failure concurrent with the main indication for heart transplantation $[5,6]$. Over the last years, substantial studies have focused on the etiology and development of DCM, whereas the exact cause of DCM was still not understood. Increasing evidence supports several cytokines implicated in the inflammatory, and immune responses are participating in the pathological process of DCM even congestive heart failure $[7,8]$. It has been delineated that the gene polymorphisms of proinflammatory cytokines such as interleukin- (IL-) 6 and tumor necrosis factor- $\alpha$ (TNF- $\alpha$ ) are associated with the susceptibility and prognosis of DCM or heart failure [7-9].

Interleukin-31 (IL-31) is a novelly detected proinflammatory cytokine belonging to gp130/IL-6 cytokine family which includes IL-6, IL-11, IL-27, oncostatin M, cardiotrophin-1, cardiotrophin-like cytokine, and leukemia inhibitory factor $[10,11]$. It is produced principally by the activated $\mathrm{CD}^{+}{ }^{+} \mathrm{T}$ cells, especially when cells are skewed toward a Th2 phenotype [10, 12]. Distinguished with gp130 family, it acts through a heterodimeric receptor consisting of IL31RA which is gp130-like receptor and oncostatin receptor (OSMR) [10]. As previously reported, IL-31 significantly 
TABLE 1: Information about PCR-RFLP in DCM and control groups.

\begin{tabular}{|c|c|c|c|c|c|c|}
\hline SNP ID & Primer sequence & $\begin{array}{l}\text { Major/minor } \\
\text { gene }\end{array}$ & $\begin{array}{l}\text { Product } \\
\text { (bp) }\end{array}$ & $\begin{array}{c}\text { Annealing } \\
\text { temperature } \\
\left({ }^{\circ} \mathrm{C}\right)\end{array}$ & $\begin{array}{l}\text { Restriction } \\
\text { enzyme }\end{array}$ & Allele (bp) \\
\hline \multirow{2}{*}{ rs4758680 } & F:5'-GATCACCCGGACTCAAAACGTG-3' & $\mathrm{C} / \mathrm{A}$ & 263 & 60 & MboII & A (263) \\
\hline & R: 5'-TTGTGCAAACCACACCTCTTCG-3’ & - & - & - & - & $\mathrm{C}(210+53)$ \\
\hline \multirow{2}{*}{ rs7977932 } & F:5'-GGTCAGTGTTGGGTTTGCAATG-3' & $\mathrm{C} / \mathrm{G}$ & 121 & 60 & ScrFI & $\mathrm{G}(74+57)$ \\
\hline & R:5'-TTGGTGATGGCACAGCCTCATA-3' & - & - & - & - & C (131) \\
\hline
\end{tabular}

stimulated the secretion of proinflammatory cytokines, such as IL-6 from monocytes and macrophages [13] and human colonic subepithelial myofibroblasts [14]. IL-6 could induce a hypertrophic response in myocytes [15], and TNF- $\alpha$ could trigger the left ventricular dilation [16]. In addition, the available data exhibited that IL-31 contributed to atopic dermatitis [17, 18], nonatopic eczema [19], systemic lupus erythematosus (SLE) [20], asthma [21], inflammatory bowel disease (IBD) [14], familial primary cutaneous amyloidosis [22], Kawasaki disease [23], hepatitis B virus liver failure [24], and allergic rhinitis [25]. These observations imply that IL-31 may contribute to the pathogenesis of DCM via cytokine modulation of immune response.

However, thus far, no study on the correlation between $I L-31$ and DCM was reported. Therefore, we conducted the pilot study to clarify the role of $I L-31$ in DCM patients in a Chinese population.

\section{Materials and Methods}

2.1. Study Subjects. The case group contained 331 subjects (male/female: $214 / 117$, mean age: $50.16 \pm 14.01$ years) diagnosed as DCM recruited from the West China Hospital from June 2002 to October 2015. Since the median of the left ventricular ejection fraction (LVEF) among DCM patients was 30\%, DCM patients were divided into two groups ( $\mathrm{LVEF}<30 \%$ versus $\mathrm{LVEF} \geq 30 \%$ ) in SNP-stratified analysis. The diagnosis of DCM was made in consistent with the criteria established by the World Health Organization/ International Society and Federation of Cardiology Task Force on the Classification of Cardiomyopathies in 1995 (before 2006) and the scientific statement on the definitions and classification of cardiomyopathies proposed by the American Heart Association in 2006 (after 2006) [2, 26]. Meanwhile, for comparison, we recruited the control group from a routine health survey, and finally, 493 healthy unrelated individuals (male/female: 312/181, mean age: $49.15 \pm 8.82$ years) were consecutively enrolled. The patients with hypertension, coronary heart disease, cardiac valve disease, tachyarrhythmia, acute viral myocarditis, heavy alcohol intake, skeletal myopathies, systemic diseases of putative autoimmune origin, diabetes, and obesity or insulin resistance were excluded from the study. Written informed consents were obtained from all included subjects, sequentially, and $10 \mathrm{~mL}$ of peripheral venous blood was drawn from each of the DCM patient and control subjects. The present study was approved by the hospital ethics committee.
2.2. Extraction of DNA and Genotyping. Genomic DNA was extracted from $200 \mu \mathrm{l}$ EDTA-anticoagulated peripheral blood sample with a DNA isolation kit (BioTeke, Peking, China) as the manufacturer's direction. DNA was stably stored at $-20^{\circ} \mathrm{C}$ until assayed. Genotyping of the $I L-31$ gene polymorphism was conducted by polymerase chain reactionrestriction fragment length polymorphism (PCR-RFLP). We designed the PCR primers with software Primer 3 (http://bioinfo.ut.ee/primer3-0.4.0/primer3/) [27] as shown in Table 1 . The $10 \mu \mathrm{l}$ PCR reaction system was consisted of $1.0 \mu \mathrm{l}$ DNA and $5 \mu \mathrm{l} 2 \times$ Power Taq PCR Master Mix (BioTeke, Peking, China), forward and reverse primer $0.1 \mu \mathrm{l}$, respectively, and reserved volume was made up to $10 \mu \mathrm{l}$ by sterilized water. The PCR condition was designed as $95^{\circ} \mathrm{C}$ for $4 \mathrm{~min}$ firstly, then 33 cycles at $95^{\circ} \mathrm{C}$ for $30 \mathrm{~s}$, $60^{\circ} \mathrm{C}$ for $30 \mathrm{~s}$, and $72^{\circ} \mathrm{C}$ for $30 \mathrm{~s}$, and finally, $72^{\circ} \mathrm{C}$ for $10 \mathrm{~min}$. Furthermore, the PCR products were digested in $37^{\circ} \mathrm{C}$ stable incubation by distinguished restriction enzyme MboII (New England Biolabs, Peking, China) for 30 minutes of rs4758680 and ScrFI (New England Biolabs, Peking, China) for 2 hours of rs7977932 as shown in Table 1, separately. Ultimately, the results were visually analyzed by $6 \%$ polyacrylamide gels in silver staining. To verify the genotyping results, DNA sequencing was performed in about $20 \%$ PCR-amplified DNA samples randomly.

2.3. $m R N A$ Isolation, Reverse Transcription, and Quantitative Real-Time PCR ( $q P C R)$. Quantitative real-time PCR of IL-31 was conducted in 41 DCM patients and 49 controls. Total RNA was isolated from white blood cells (WBCs) with TRIzol reagent (Invitrogen, Karlsruhe, Germany) and then was reverse-transcribed into cDNA, using the Bioneer kit (R\&D Center, Korea) following the manufacturer's protocols. Actin was chosen as a reference parameter to normalize the data. The sense and anti-sense primers for IL-31 and Actin were 5'-CTCACTCAGGCCCCTCG AC-3' and 5'-GTCGTAGTAAACGGACGGGC-3', 5'-TGA CGTGGACATCCGCAAAG- $3^{\prime}$ and $5{ }^{\prime}$-CTGGAAGGTGGA CAGCGAGG-3', respectively.

The qPCR settings in triplicate carried on a MasterCycler realplex ${ }^{4}$ (Eppendorf, Wesseling-Berzforf, Germany) using SYBR Green were as follows: an initial activation step of $10 \mathrm{~min}$ at $95^{\circ} \mathrm{C}$, subsequently by two-step cycling for 25 times: denaturation of $15 \mathrm{~s}$ at $94^{\circ} \mathrm{C}$, and annealing and extension of $20 \mathrm{~s}$ at $60^{\circ} \mathrm{C}$. Melting curve was added to check the amplification specificity. $2^{-\triangle c t}$ method was used to calculate the mRNA expression levels [28]. 
TABLE 2: Baseline characteristics of the DCM patients and the controls.

\begin{tabular}{lccc}
\hline Variables & DCM patients $(n=331)$ & Controls $(n=493)$ & $P$ value \\
\hline Age (years) & $52(43-60)$ & $50(42-57)$ & 0.022 \\
Gender (male/female) & $214 / 117$ & $312 / 181$ & 0.689 \\
SBP $(\mathrm{mmHg})$ & $116(109-123)$ & $121(114-127)$ & $<0.001$ \\
DBP $(\mathrm{mmHg})$ & $73(68-77)$ & $77(72-81)$ & $<0.001$ \\
NYHA & II: 62; III: $210 ;$ IV: 59 & I: $395 ;$ II: 98 & $<0.001$ \\
LVEDD $(\mathrm{mm})$ & $67(62-73)$ & $46(44-49)$ & $<2(58-66)$ \\
LVEF $(\%)$ & $30(25-37)$ & $95(83-108)$ & $<0.001$ \\
BNP $(\mathrm{pg} / \mathrm{ml})$ & $2787(1600-3836)$ & $<0.001$ \\
\hline
\end{tabular}

Data are exhibited as the median \pm interquartile range(IQR: Q25\%-Q75\%) or number; DCM: dilated cardiomyopathy; SBP: systolic blood pressure; DBP: diastolic blood pressure; NYHA: New York Heart Association; LVEDD: left ventricular end-diastolic diameter; LVEF: left ventricular ejection fraction; BNP: brain natriuretic peptide.

2.4. Patients' Clinical Characteristics and Follow-Up. One hundred and fifty-nine DCM patients who have reserved contact information were brought into follow-up plan every three months until September 13, 2016. Basic clinical materials of enrolled DCM patients were obtained from the medical records (age, gender, etc.) and echocardiographic measurement using a S5-1 broadband phased-array transducer $(1-5 \mathrm{MHz})$. According to the recommendations of the American Society of Echocardiography, we conducted a comprehensive 2D and Doppler echocardiography. The echocardiographic indicators such as the left ventricular end-diastolic diameter (LVEDD) were calculated with $\mathrm{M}$ mode echocardiography with the left parasternal window while the left ventricular ejection fraction (LVEF) was accessed by apical two- and four-chamber views with the modified Simpson rule. The follow-up end point was patient's death because of heart failure or sudden cardiac events. A blind manner about patient's genetic status was applied during clinical follow-up.

2.5. Statistical Analysis. Quantitative variables were presented as median and interquartile range (IQR: Q25\%-Q75\%) and categorical variables as number of observations. Normality was tested using Shapiro-Wilk's test for normality. Differences between two independent samples for continuous data were analyzed using the Mann-Whitney $U$ test since the distributions were different from normal. For categorical variables, statistical analysis was based on Pearson's chi-square test.

The allelic and genotype frequencies were obtained by number counting. The differences of genotypes between the DCM and control groups including codominant, dominant, recessive, and overdominant genetic models were analyzed by using SNPStats online program; meanwhile, odds ratio and $95 \%$ confidence intervals were obtained accordingly [29].

Allelic association and Hardy-Weinberg equilibrium were assessed with chi-square test. IL-31 WBC mRNA expression level was compared using the Mann-Whitney test (for two independent groups) and Kruskal-Wallis $\mathrm{H}$ test with multiple comparisons post hoc tests according to the results from Shapiro-Wilk's test for normality.

Kaplan-Meier curve and Cox proportional hazard models were applied to evaluate the role of IL-31 SNPs on prognosis in DCM patients. $P$ value less than 0.05 was regarded as statistically significant.

\section{Results}

3.1. Baseline Characteristics of DCM Patients and the Controls. As shown in Table 2, between the DCM patients and the controls, gender did not exhibit statistically significant differences $(P=0.689)$. Compared to controls, DCM patients were older $(P=0.022)$ and had lower systolic blood pressure (SBP), diastolic blood pressure (DBP), left ventricular ejection fraction (LVEF), higher left ventricular end-diastolic diameter (LVEDD), and brain natriuretic peptide (BNP) $(P<0.001$, resp.) as well as more severe NYHA functional class $(P<0.001)$. All DCM patients accepted medication treatment according to the clinical guidelines for DCM and heart failure.

3.2. Associations between IL-31 SNPs and Susceptibility for DCM and DCM Patients' Characteristics. The gene polymorphisms of $I L-31$ rs4758680 and rs7977932 were identified to compare the allelic and genotype frequencies of 331 DCM patients to 493 controls through direct counting. About 20\% PCR-amplified DNA samples were randomly evaluated by DNA sequencing, and the PCR-RFLP results were manifested as $100 \%$ accurate. The distribution of both rs4758680 and rs7977932 alleles in control groups was line with the postulation of Hardy-Weinberg equilibrium $\left(\chi^{2}=1.876, P=0.17\right.$ for $\mathrm{rs} 4758680 ; \chi^{2}=0.004, P=0.95$ for rs7977932).

As shown in Table 3, the strikingly statistical difference was discovered at rs4758680. The allele frequency of $\mathrm{C}$ of SNP rs4758680 in DCM patients was significantly elevated compared with that in controls (89.6\% versus $84.8 \%$ ); in contrast, the allele A frequency was declined (10.4\% versus $15.2 \%, P=0.005, \mathrm{OR}=0.65,95 \% \mathrm{CI}=0.48-0.88)$ in case group. In codominant model, the frequencies of the CC, CA, and AA genotypes of rs4758680 were $83.1 \%, 13.0 \%$, and $3.9 \%$ in cases and were $73.2 \%, 23.1 \%$, and $3.6 \%$ in controls, respectively. The differences among genotype frequencies were statistically significant $(P=0.001)$. In dominant model, compared with CC genotype, a notably decreased DCM risk was related with $\mathrm{CA} / \mathrm{AA}$ genotypes $(P<0.001$, 


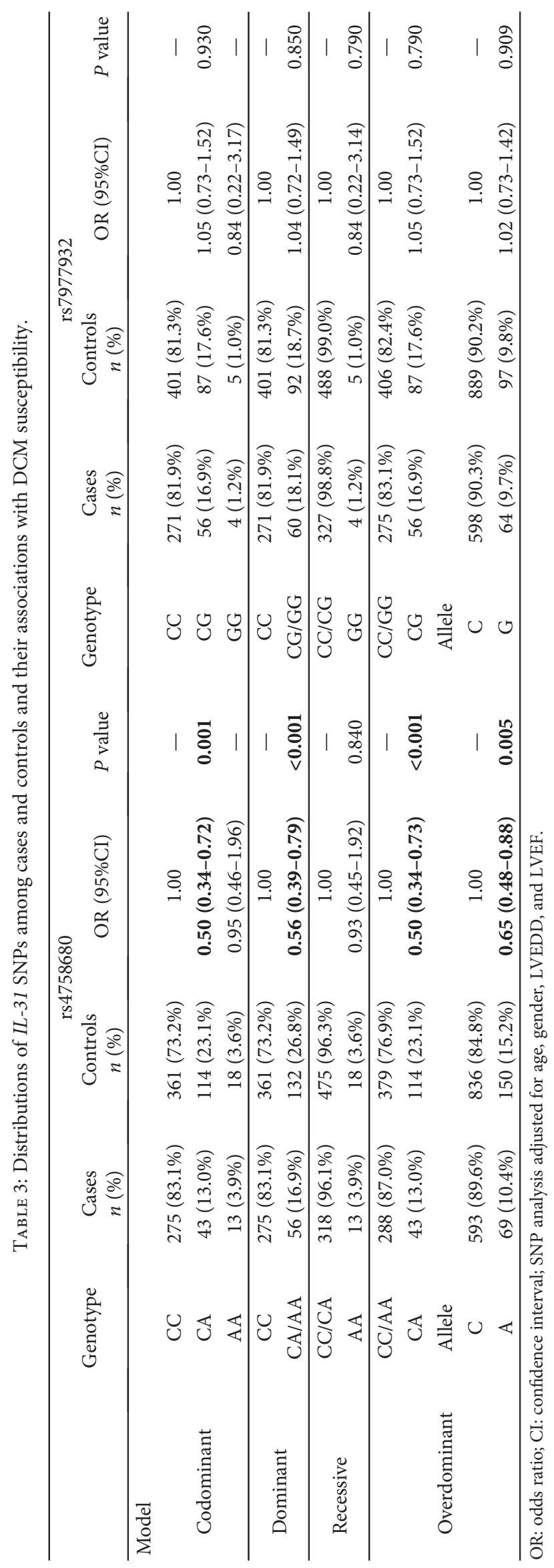


$\mathrm{OR}=0.56,95 \% \mathrm{CI}=0.39-0.79)$. Subjects with heterozygous genotype (CA genotype) of rs 4758680 had distinctly decreased risks for DCM compared with the CC/AA genotypes in overdominant model $(P<0.001, \mathrm{OR}=0.50,95 \% \mathrm{CI}=0.34-$ $0.73)$. As shown in Table 3 , there was no significant difference described between DCM patients and controls in rs7977932 gene polymorphism analysis. To provide insights into the effects of $I L-31$ SNPs on DCM, we exhibited the stratified analyses among 331 DCM patients. After adjusted by age and gender, the association between rs4758680 of $I L-31$ and LVEF was shown in Table 4 which revealed that the heterozygote CA was the protect factor for DCM patients whose LVEF was $<30 \%$ compared with those LVEF was $\geq 30 \% \quad(P=0.042)$. There were no statistically significant differences between the two SNPs of $I L-31$ (rs4758680 and rs7977932) and LVEDD.

3.3. IL-31 WBC mRNA Expression Level. The median and IQR of $2^{-\triangle \mathrm{ct}}$ result among $41 \mathrm{DCM}$ patients were 0.072 (0.044-0.144), while it was 0.036 (0.020-0.052) among 49 controls, and the difference for IL-31 WBC mRNA expression between DCM group $(n=41)$ and control group $(n=49)$ was statistically significant $(P<0.001)$ as shown in Figure 1(a). To probe into the functional influence of the IL-31 (rs4758680) genotype polymorphism (CC, CA, and AA genotypes) on the IL-31 WBC mRNA expression, the quantitative IL-31 mRNA results among different genotypes were analyzed. The IL-31 mRNA results for CA/AA genotypes in DCM $(n=21)$ and controls $(n=22)$ were 0.087 (0.044-0.223) and $0.038(0.021-0.050)$, and those for CC genotype in DCM $(n=20)$ and controls $(n=27)$ were 0.065 (0.044-0.124) and $0.033(0.018-0.065)$. As Figure 1(b) delineated, the $P$ value was less than 0.001 for KruskalWallis $\mathrm{H}$ test and the results of multiple comparisons post hoc tests revealed that there were statistically significant differences for IL-31 mRNA level between CA/AA genotypes in DCM and CA/AA genotypes in controls, CC genotype in DCM and CA/AA genotypes in controls, CA/AA genotypes in DCM and CC genotype in controls, and CC genotype in DCM and CC genotype in controls $(P<0.001$, resp.). There were no statistically significant differences between CA/AA genotypes and CC genotype in DCM, as well as in controls $(P=0.191,0.389$, resp.).

3.4. Survival Analysis of IL-31 Genotypes in DCM Patients. The prognosis of DCM associated with two SNPs of IL-31 gene was carried out by survival analysis. 159 DCM patients (mean age, $51.03 \pm 13.43$ years; male/female, 107/52) were tracked for a mean period of three months. 20 DCM patients were lost during the follow-up. During the follow-up, all included patients accepted consecutive medication treatment and none underwent heart transplantation.

When ending up the follow-up, 104 (65.4\%) DCM patients died ascribable to cardiac events. The baseline characteristic differences between 104 dead DCM patients and 35 survival DCM patients were described in Supplementary Table available online at https://doi.org/10.1155/2017/4191365, and there were no statistically significant differences between the two analyzed groups for gender, SBP, DBP, and LVEDD
$(P=0.251,0.789,0.431$, and 0.817 , resp.). By contrast, dead DCM patients were younger $(P=0.036)$ and had worse NYHA functional class $(P<0.001)$, lower LVEF $(P=0.025)$, and higher $\operatorname{BNP}(P=0.020)$.

Kaplan-Meier curves indicated that CC homozygote and CC/AA genotypes of $I L-31$ rs4758680 were implicated in worse prognosis for DCM patients, respectively (Logrank: $P=0.005$, Figure 2; Log-rank: $P=0.009$, Figure 3 ). Cox univariate survival analysis revealed A carriers (CA/ AA genotypes) were correlated with better prognosis compared to $\mathrm{CC}$ genotype in genetic dominant model for DCM patients $(\mathrm{HR}=0.530,95 \% \mathrm{CI}=0.337-0.834, P=0.006$, Table 5). Similarly in overdominant model of rs4758680, compared to CC and AA homozygotes, heterozygote CA accounted for better prognosis for DCM patients $(\mathrm{HR}=0.516,95 \% \mathrm{CI}=0.310-0.861, \quad P=0.011$, Table 5). After adjusting for age, gender, LVEDD, and LVEF, the associations between A carriers (CA/AA genotypes), CA heterozygote of rs4758680, and prognosis of DCM were still statistical significant for both dominant and overdominant models in multivariate Cox proportional hazard model analysis $(\mathrm{HR}=0.548,95 \% \mathrm{CI}=0.345-0.869, P=0.011$ and $\mathrm{HR}=0.503,95 \% \mathrm{CI}=0.297-0.852, P=0.011$, Table 5, resp.). There were no statistical differences between $I L-31$ rs4758680 recessive model as well as rs7977932 gene polymorphism and overall survival time in univariate and multivariate Cox proportional hazard models as shown in Table 5.

\section{Discussion}

As previously reported, although the etiology and pathogenesis of DCM were complicated, chronic inflammation might contribute to cardiac remodeling and the development of DCM [30], and abnormal immune responses were proposed to be prominent factors in the DCM process especially after myocarditis [31]. Inflammatory cytokines like IL-6 in conjunction with TNF- $\alpha$ participated in myocyte apoptosis and myofibrosis which were involved in DCM $[15,32]$.

Beyond that, the established studies validated that IL-31 as a novel inflammatory cytokine was a potent inducer of proinflammatory mediators such as IL- 6 in various cell types, including epithelial cells, colonic subepithelial myofibroblasts, PBMCs, macrophages, and eosinophils [33]. Matrix metalloproteinases (MMPs) also can be induced by IL-31 in colonic subepithelial myofibroblasts of IBD [14] and their higher serum level was involved in continuous extracellular matrix remodeling and increased collagen turnover of DCM with mildly dilated left ventricle $[34,35]$. Shen et al. revealed that inhibition of MMPs, especially MMP-2, could reduce apoptosis from TNF- $\alpha$ in cultured cardiac myocytes [36]. In the past few years, IL-31 receptor was principally found in the skin, brain, lung, skeletal muscle, ovary, testis, prostate, spleen, thymus, bone marrow, and more [10]. In coincident with IL-31 receptor distribution, IL-31 was already identified to be associated with immune-dysfunction diseases such as atopic dermatitis, SLE, and asthma.

The established studies suggested that soluble IL-31RA might expand the range of responsive cells and tissues because of the transsignaling for IL-6 [37]; meanwhile, 


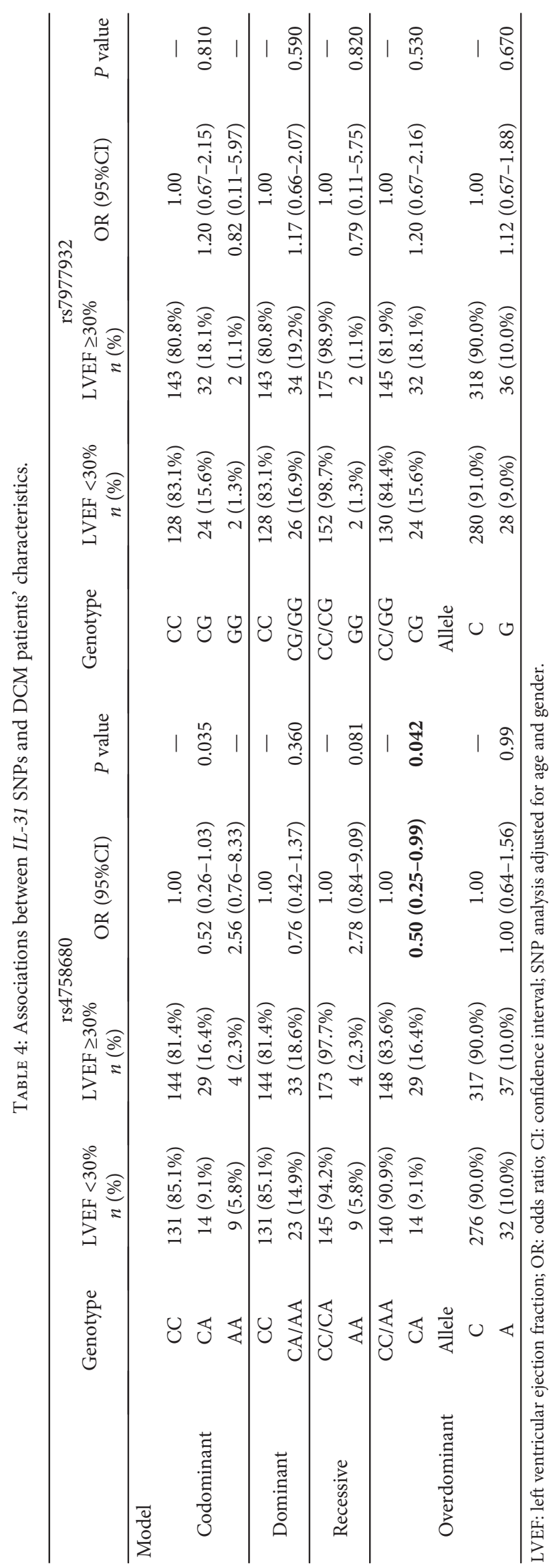




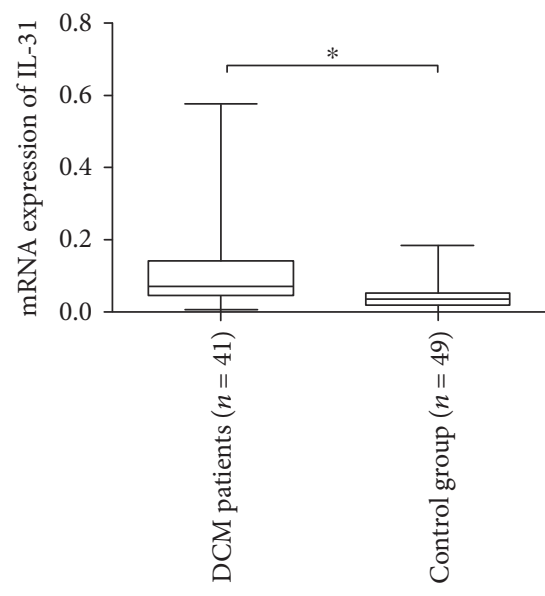

(a)

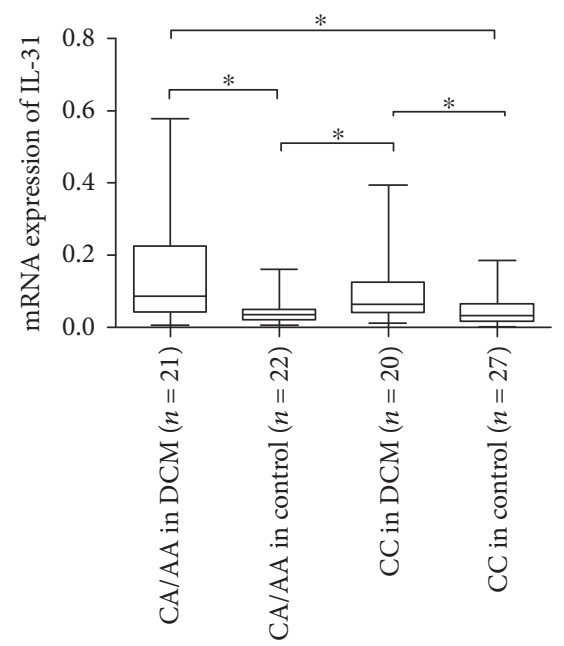

(b)

FIgURE 1: Comparsion of WBC IL-31 mRNA expression level between the DCM patients and the control group (a); comparsions of WBC IL-31 mRNA expression levels among rs4758680 different genotypes (b). The results were presented on box plots (median, IQR, range), ${ }^{*} P<0.001$.

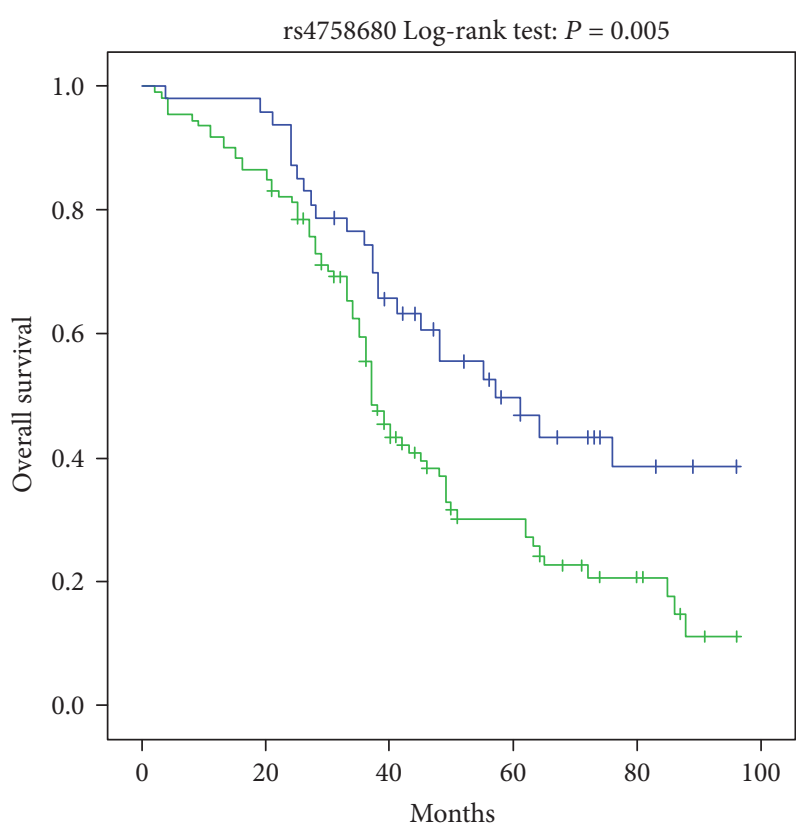

$\begin{array}{ll}\text { Genotype } & \\ \neg \mathrm{CA}+\mathrm{AA} & +\mathrm{CA}+\mathrm{AA} \text { censored } \\ \neg \mathrm{CC} & +\mathrm{CC} \text { censored }\end{array}$

FIGURE 2: Kaplan-Meier survival curves for the dominant model of IL-31 rs4758680 polymorphism.

myocarditis had overlapping loci with diabetes and SLE, suggesting that these autoimmune diseases shared genetic traits [1]. Similarly, Doria et al. identified that DCM was one of the most serious complications involved in SLE [38] and D. Y. Chen et al. have verified that the level of Th17-related cytokines (containing IL-6, TNF- $\alpha$ ) was elevated in SLE-related DCM [39]. These inferences imply a
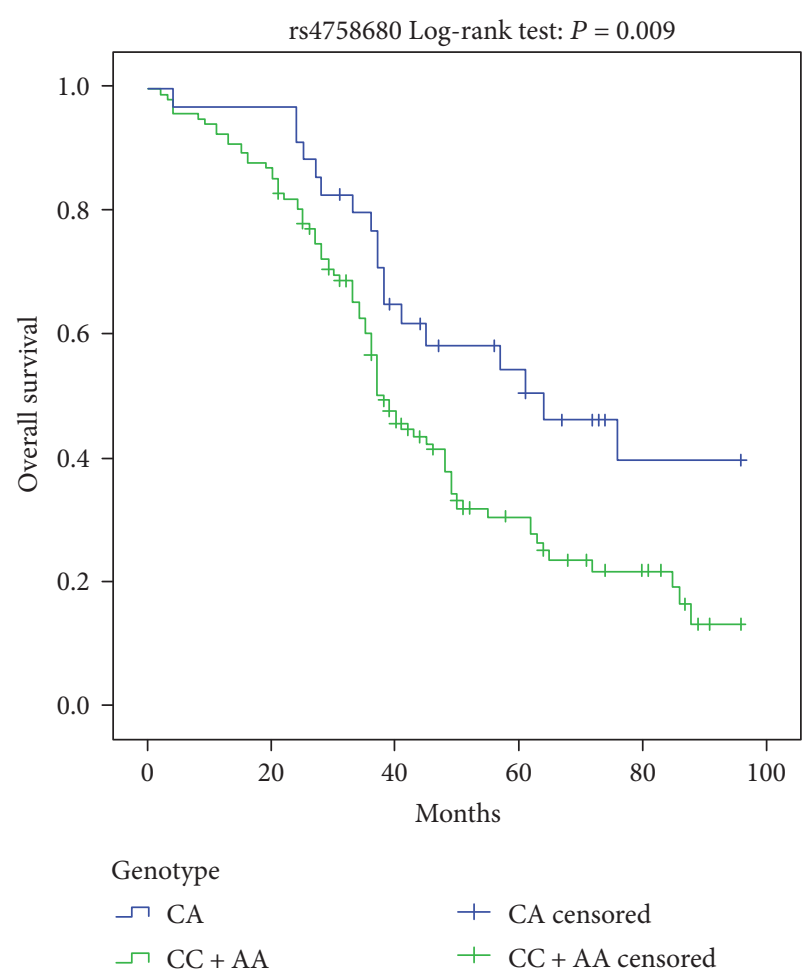

FIgURE 3: Kaplan-Meier survival curves for the overdominant model of IL-31 rs4758680 polymorphism.

possible role of IL-31 in immune response and in DCM pathogenesis process.

The present study was the first one to investigate the correlation between IL-31 and DCM in a Han Chinese population. The human IL-31 gene is located on chromosome $12 \mathrm{q} 24.31$ and encodes a protein with 164 amino acids. Both rs4758680 (C/A) and rs7977932 (C/G) are in chromosome 
TABLE 5: Associations between IL-31 SNPs and patients' overall survival.

\begin{tabular}{|c|c|c|c|c|c|c|c|}
\hline \multirow{3}{*}{ Characteristics } & \multirow{3}{*}{ Genotype } & \multicolumn{6}{|c|}{ Overall survival } \\
\hline & & \multicolumn{3}{|c|}{ Multivariate survival analysis ${ }^{\mathrm{a}}$} & \multicolumn{3}{|c|}{ Univariate survival analysis } \\
\hline & & HR & $95 \% \mathrm{CI}$ & $P$ value & HR & $95 \% \mathrm{CI}$ & $P$ value \\
\hline \multicolumn{8}{|l|}{ Model } \\
\hline \multicolumn{8}{|l|}{ rs4758680 } \\
\hline \multirow{2}{*}{ Dominant } & $\mathrm{CC}$ & 1 & - & - & 1 & - & - \\
\hline & CA/AA & 0.548 & $0.345-0.869$ & 0.011 & 0.530 & $0.337-0.834$ & 0.006 \\
\hline \multirow{2}{*}{ Recessive } & $\mathrm{CC} / \mathrm{CA}$ & 1 & - & - & 1 & - & - \\
\hline & AA & 0.868 & $0.399-1.888$ & 0.722 & 0.778 & $0.361-1.678$ & 0.522 \\
\hline \multirow{2}{*}{ Overdominant } & CC/AA & 1 & - & - & 1 & - & - \\
\hline & $\mathrm{CA}$ & 0.503 & $0.297-0.852$ & 0.011 & 0.516 & $0.310-0.861$ & 0.011 \\
\hline \multicolumn{8}{|l|}{ rs7977932 } \\
\hline \multirow{2}{*}{ Dominant } & $\mathrm{CC}$ & 1 & - & - & 1 & - & - \\
\hline & CG/GG & 1.161 & $0.666-2.024$ & 0.599 & 1.275 & $0.736-2.211$ & 0.386 \\
\hline \multirow{2}{*}{ Recessive } & $\mathrm{CC} / \mathrm{CG}$ & 1 & - & - & 1 & - & - \\
\hline & GG & 3.739 & $0.862-16.218$ & 0.078 & 3.482 & $0.841-14.414$ & 0.085 \\
\hline \multirow{2}{*}{ Overdominant } & $\mathrm{CC} / \mathrm{GG}$ & 1 & - & - & 1 & - & - \\
\hline & CG & 1.096 & $0.619-1.943$ & 0.753 & 1.211 & $0.687-2.133$ & 0.508 \\
\hline
\end{tabular}

${ }^{a}$ Multivariate survival analysis adjusted for age, gender, LVEDD, and LVEF.

12 intron region of Homo sapiens which have been implicated in SLE and AD [19, 20, 40].

Our results showed that genotype frequencies in the codominant, dominant, and overdominant models of rs4758680 were associated with DCM susceptibility. The C allele frequency of $\mathrm{rs} 4758680$ (C/A) in DCM was elevated, whereas the A allele was declined. We manifested that the $\mathrm{C}$ allele was the main predisposing factor and A carriers (CA/AA genotypes) were the protect factors for DCM especially in genetic dominant model. The CC genotype frequency of rs4758680 of $I L-31$ was also relevance with DCM worse prognosis in Kaplan-Meier curve and Cox proportional hazard models. Moreover, in accordance with SNP results of rs4758680, the IL-31 WBC mRNA expression level was overtly elevated in DCM group, and the WBC mRNA expression levels of CC and CA/AA genotypes in DCM patients were higher than those in control group. As previously reported, IL-31 acted through the receptor complex of IL-31RA which is gp130-like receptor and OSMR- $\beta$. gp130 has been shown to mediate the cardiotrophin-1 (CT-1) in the heart that resulted in LV hypertrophy [41]. OSMR- $\beta$ has been proved as increasing expression trend in DCM patients [42] and signaling in myocardium that result in loss of sarcomere elements and cardiac fibroblast in mouse cardiac fibroblasts [43]. Kunsleben et al. delineated that the calcium influx was induced by IL-31 in eosinophils mainly through OSMR mediating, which prompt IL-31 may affect the myocardial contraction [44]. Hence, we concluded that rs4758680 of $I L-31$ SNPs played a pathogenic role in DCM patients by facilitating IL-31 protein production. In our study, the number of WBC mRNA samples was not large enough, especially the number of AA genotype was only three cases and two cases in DCM and controls, respectively. The precise and intricate mechanisms for protein expression were still unclear, and much more studies will be indispensable. By contrast, the genotype frequency of rs7977932 was absence in the DCM susceptibility. As previously reported, rs7977932 (C/G) genotype polymorphism of $I L-31$ was implicated in SLE and AD [20, 40]. The difference of inclusive quantity or disease essence between our study in DCM and the previous one in SLE may account for the inconsistency of rs7977932 effect.

\section{Conclusions}

In conclusion, IL-31 as scratch factor was a research hot spot before we firstly revealed that rs4758680 (C/A) of $I L-31$ was associated with the susceptibility of DCM in the Chinese Han people although larger sample sizes of $I L-31$ SNP would be necessary to confirm our findings; moreover, CC genotype was implicated in the worse prognosis in DCM group. Even so, plasma IL-31 protein level and the underlying mechanisms were lack in our study; besides, more SNPs of $I L-31$ with DCM susceptibility and prognosis in a variety of ethnic populations need to be investigated in future studies.

\section{Conflicts of Interest}

The authors declare that there is no conflict of interests regarding the publication of this paper.

\section{Authors' Contributions}

Huizi Song and Ying Peng contributed equally to this work.

\section{Acknowledgments}

This work was supported by the National Natural Science Foundation of China (no. 81270289 and no. 81300170). 


\section{References}

[1] J. L. Jefferies and J. A. Towbin, "Dilated cardiomyopathy," Lancet, vol. 375, no. 9716, pp. 752-762, 2010.

[2] P. Richardson, W. McKenna, M. Bristow et al., "Report of the 1995 World Health Organization/International Society and Federation of Cardiology Task Force on the definition and classification of cardiomyopathies," Circulation, vol. 93, no. 5, pp. 841-842, 1996.

[3] M. B. Codd, D. D. Sugrue, B. J. Gersh, and L. J. Melton, "Epidemiology of idiopathic dilated and hypertrophic cardiomyopathy. A population-based study in Olmsted County, Minnesota, 1975-1984," Circulation, vol. 80, no. 3, pp. 564$572,1989$.

[4] M. R. Taylor, E. Carniel, and L. Mestroni, "Cardiomyopathy, familial dilated," Orphanet Journal of Rare Diseases, vol. 1, no. 1, p. 27,2006

[5] O. P. Guttmann, S. A. Mohiddin, and P. M. Elliott, "Almanac 2014: cardiomyopathies," Heart, vol. 100, no. 10, pp. 756-764, 2014.

[6] M. J. Everly, "Cardiac transplantation in the United States: an analysis of the UNOS registry," Clinical Transplants, pp. 35-43, 2008.

[7] D. L. Mann and J. B. Young, "Basic mechanisms in congestive heart failure. Recognizing the role of proinflammatory cytokines," Chest, vol. 105, no. 3, pp. 897-904, 1994.

[8] P. Aukrust, T. Ueland, E. Lien et al., "Cytokine network in congestive heart failure secondary to ischemic or idiopathic dilated cardiomyopathy," The American Journal of Cardiology, vol. 83, no. 3, pp. 376-382, 1999.

[9] S. Adamopoulos, F. Kolokathis, A. Gkouziouta et al., "Cytokine gene polymorphisms are associated with markers of disease severity and prognosis in patients with idiopathic dilated cardiomyopathy," Cytokine, vol. 54, no. 1, pp. 6873, 2011.

[10] S. R. Dillon, C. Sprecher, A. Hammond et al., "Interleukin 31, a cytokine produced by activated $\mathrm{T}$ cells, induces dermatitis in mice," Nature Immunology, vol. 5, no. 7, pp. 752-760, 2004.

[11] S. Pflanz, L. Hibbert, J. Mattson et al., "WSX-1 and glycoprotein 130 constitute a signal-transducing receptor for IL-27," Journal of Immunology, vol. 172, no. 4, pp. 2225-2231, 2004.

[12] E. Sonkoly, A. Muller, A. I. Lauerma et al., "IL-31: a new link between $\mathrm{T}$ cells and pruritus in atopic skin inflammation," The Journal of Allergy and Clinical Immunology, vol. 117, no. 2, pp. 411-417, 2006.

[13] S. Kasraie, M. Niebuhr, and T. Werfel, "Interleukin (IL)-31 induces pro-inflammatory cytokines in human monocytes and macrophages following stimulation with staphylococcal exotoxins," Allergy, vol. 65, no. 6, pp. 712-721, 2010.

[14] Y. Yagi, A. Andoh, A. Nishida et al., "Interleukin-31 stimulates production of inflammatory mediators from human colonic subepithelial myofibroblasts," International Journal of Molecular Medicine, vol. 19, no. 6, pp. 941-946, 2007.

[15] S. P. Janssen, G. Gayan-Ramirez, A. Van den Bergh et al., "Interleukin-6 causes myocardial failure and skeletal muscle atrophy in rats," Circulation, vol. 111, no. 8, pp. 996-1005, 2005.

[16] W. S. Bradham, B. Bozkurt, H. Gunasinghe, D. Mann, and F. G. Spinale, "Tumor necrosis factor-alpha and myocardial remodeling in progression of heart failure: a current perspective," Cardiovascular Research, vol. 53, no. 4, pp. 822830, 2002.
[17] A. Kato, E. Fujii, T. Watanabe et al., "Distribution of IL-31 and its receptor expressing cells in skin of atopic dermatitis," Journal of Dermatological Science, vol. 74, no. 3, pp. 229235, 2014.

[18] M. M. Neis, B. Peters, A. Dreuw et al., "Enhanced expression levels of IL-31 correlate with IL-4 and IL-13 in atopic and allergic contact dermatitis," The Journal of Allergy and Clinical Immunology, vol. 118, no. 4, pp. 930-937, 2006.

[19] F. Schulz, I. Marenholz, R. Fölster-Holst et al., "A common haplotype of the IL-31 gene influencing gene expression is associated with nonatopic eczema," The Journal of Allergy and Clinical Immunology, vol. 120, no. 5, pp. 1097-1102, 2007.

[20] H. T. Huang, J. M. Chen, J. Guo, Y. Lan, and Y. S. Wei, "The association of interleukin-31 polymorphisms with interleukin-31 serum levels and risk of systemic lupus erythematosus," Rheumatology International, vol. 36, no. 6, pp. 799-805, 2016.

[21] J. I. Yu, W. C. Han, K. J. Yun, H. B. Moon, G. J. Oh, and S. C. Chae, "Identifying polymorphisms in IL-31 and their association with susceptibility to asthma," Korean Journal of Pathology, vol. 46, no. 2, pp. 162-168, 2012.

[22] Y. M. Shiao, H. J. Chung, C. C. Chen et al., "MCP-1 as an effector of IL-31 signaling in familial primary cutaneous amyloidosis," The Journal of Investigative Dermatology, vol. 133, no. 5, pp. 1375-1378, 2013.

[23] W. N. Tseng, M. H. Lo, M. M. Guo, K. S. Hsieh, W. C. Chang, and H. C. Kuo, "IL-31 associated with coronary artery lesion formation in Kawasaki disease," PloS One, vol. 9, no. 8, article e105195, 2014.

[24] X. Yu, R. Guo, D. Ming et al., "The transforming growth factor $\beta 1 /$ interleukin-31 pathway is upregulated in patients with hepatitis B virus-related acute-on-chronic liver failure and is associated with disease severity and survival," Clinical and Vaccine Immunology, vol. 22, no. 5, pp. 484-492, 2015.

[25] M. Okano, T. Fujiwara, T. Higaki et al., "Characterization of pollen antigen-induced IL-31 production by PBMCs in patients with allergic rhinitis," The Journal of Allergy and Clinical Immunology, vol. 127, no. 1, pp. 277-279, 2011, 279.e1-11.

[26] B. J. Maron, J. A. Towbin, G. Thiene et al., "Contemporary definitions and classification of the cardiomyopathies: an American Heart Association scientific statement from the council on clinical cardiology, heart failure and transplantation committee; quality of care and outcomes research and functional genomics and translational biology interdisciplinary working groups; and council on epidemiology and prevention," Circulation, vol. 113, no. 14, pp. 1807-1816, 2006.

[27] E. F. Vieux, P. Y. Kwok, and R. D. Miller, "Primer design for PCR and sequencing in high-throughput analysis of SNPs," BioTechniques, vol. 32, Supplement, pp. 28-30, 2002.

[28] T. D. Schmittgen and K. J. Livak, "Analyzing real-time PCR data by the comparative $\mathrm{C}(\mathrm{T})$ method," Nature Protocols, vol. 3, no. 6, pp. 1101-1108, 2008.

[29] X. Solé, E. Guinó, J. Valls, R. Iniesta, and V. Moreno, "SNPStats: a web tool for the analysis of association studies," Bioinformatics, vol. 22, no. 15, pp. 1928-1929, 2006.

[30] S. Sagar, P. P. Liu, and L. T. Cooper, "Myocarditis," Lancet, vol. 379, no. 9817, pp. 738-747, 2012.

[31] F. Schwarz, G. Mall, H. Zebe et al., "Quantitative morphologic findings of the myocardium in idiopathic dilated cardiomyopathy," The American Journal of Cardiology, vol. 51, no. 3, pp. 501-506, 1983. 
[32] K. A. Krown, M. T. Page, C. Nguyen et al., "Tumor necrosis factor alpha-induced apoptosis in cardiac myocytes. Involvement of the sphingolipid signaling cascade in cardiac cell death," The Journal of Clinical Investigation, vol. 98, no. 12, pp. 2854-2865, 1996.

[33] J. Horejs-Hoeck, H. Schwarz, S. Lamprecht et al., "Dendritic cells activated by IFN- $\gamma /$ STAT1 express IL-31 receptor and release proinflammatory mediators upon IL-31 treatment," Journal of Immunology, vol. 188, no. 11, pp. 5319-5326, 2012.

[34] P. Rubiś, S. Wiśniowska-Śmialek, E. Wypasek et al., "Fibrosis of extracellular matrix is related to the duration of the disease but is unrelated to the dynamics of collagen metabolism in dilated cardiomyopathy," Inflammation Research, vol. 65, no. 12, pp. 941-949, 2016.

[35] F. Picard, M. Brehm, M. Fassbach et al., "Increased cardiac mRNA expression of matrix metalloproteinase-1 (MMP-1) and its inhibitor (TIMP-1) in DCM patients," Clinical Research in Cardiology, vol. 95, no. 5, pp. 261-269, 2006.

[36] J. Shen, D. O'Brien, and Y. Xu, "Matrix metalloproteinase-2 contributes to tumor necrosis factor alpha induced apoptosis in cultured rat cardiac myocytes," Biochemical and Biophysical Research Communications, vol. 347, no. 4, pp. 10111020, 2006.

[37] C. Cornelissen, J. Lüscher-Firzlaff, J. M. Baron, and B. Lüscher, "Signaling by IL-31 and functional consequences," European Journal of Cell Biology, vol. 91, no. 6-7, pp. 552-566, 2012.

[38] A. Doria, L. Iaccarino, P. Sarzi-Puttini, F. Atzeni, M. Turriel, and M. Petri, "Cardiac involvement in systemic lupus erythematosus," Lupus, vol. 14, no. 9, pp. 683-686, 2005.

[39] D. Y. Chen, Y. M. Chen, B. S. Tzang, J. L. Lan, and T. C. Hsu, "Th17-related cytokines in systemic lupus erythematosus patients with dilated cardiomyopathies: a possible linkage to parvovirus B19 infection," PloS One, vol. 9, no. 12, article e113889, 2014.

[40] M. Sokołowska-Wojdyło, J. Gleń, M. Zabłotna et al., “Association of distinct IL-31 polymorphisms with pruritus and severity of atopic dermatitis," Journal of the European Academy of Dermatology and Venereology, vol. 27, no. 5, pp. 662-664, 2013.

[41] M. Saito, K. Yoshida, M. Hibi, T. Taga, and T. Kishimoto, "Molecular cloning of a murine IL-6 receptor-associated signal transducer, gp130, and its regulated expression in vivo," Journal of Immunology, vol. 148, no. 12, pp. 4066-4071, 1992.

[42] T. Kubin, J. Pöling, S. Kostin et al., "Oncostatin M is a major mediator of cardiomyocyte dedifferentiation and remodeling," Cell Stem Cell, vol. 9, no. 5, pp. 420-432, 2011.

[43] P. J. Lafontant, A. R. Burns, E. Donnachie, S. B. Haudek, C. W. Smith, and M. L. Entman, "Oncostatin M differentially regulates CXC chemokines in mouse cardiac fibroblasts," American Journal of Physiology. Cell Physiology, vol. 291, no. 1, pp. C18-C26, 2006.

[44] N. Kunsleben, U. Rüdrich, M. Gehring, N. Novak, A. Kapp, and U. Raap, "IL-31 induces chemotaxis, calcium mobilization, release of reactive oxygen species, and CCL26 in eosinophils, which are capable to release IL-31," The Journal of Investigative Dermatology, vol. 135, no. 7, pp. 1908-1911, 2015. 


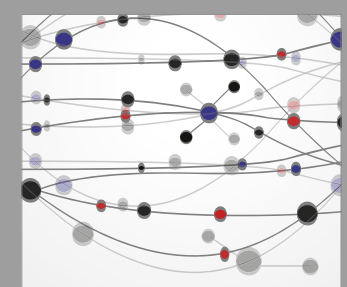

The Scientific World Journal
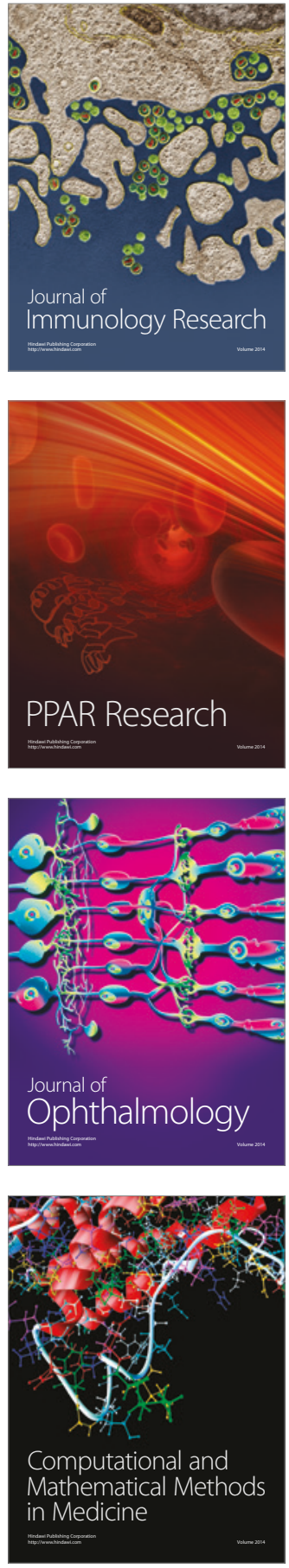

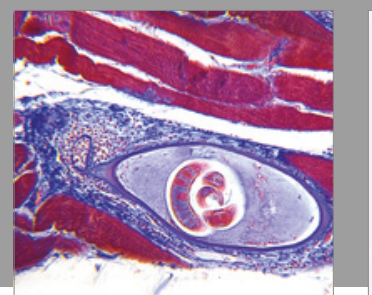

Gastroenterology Research and Practice
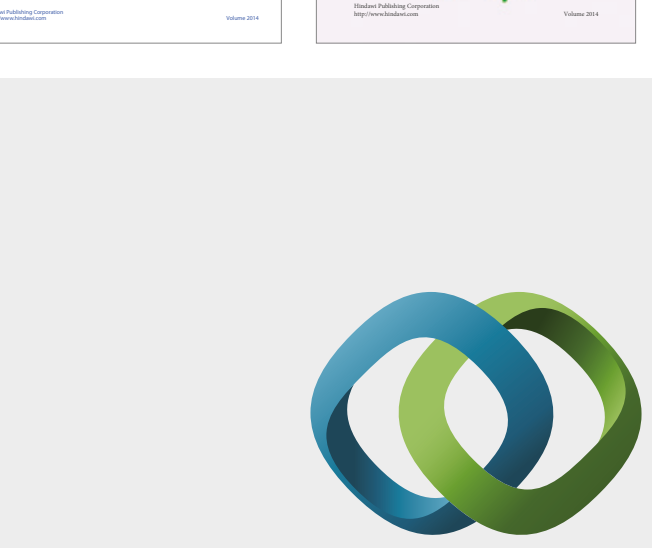

\section{Hindawi}

Submit your manuscripts at

https://www.hindawi.com
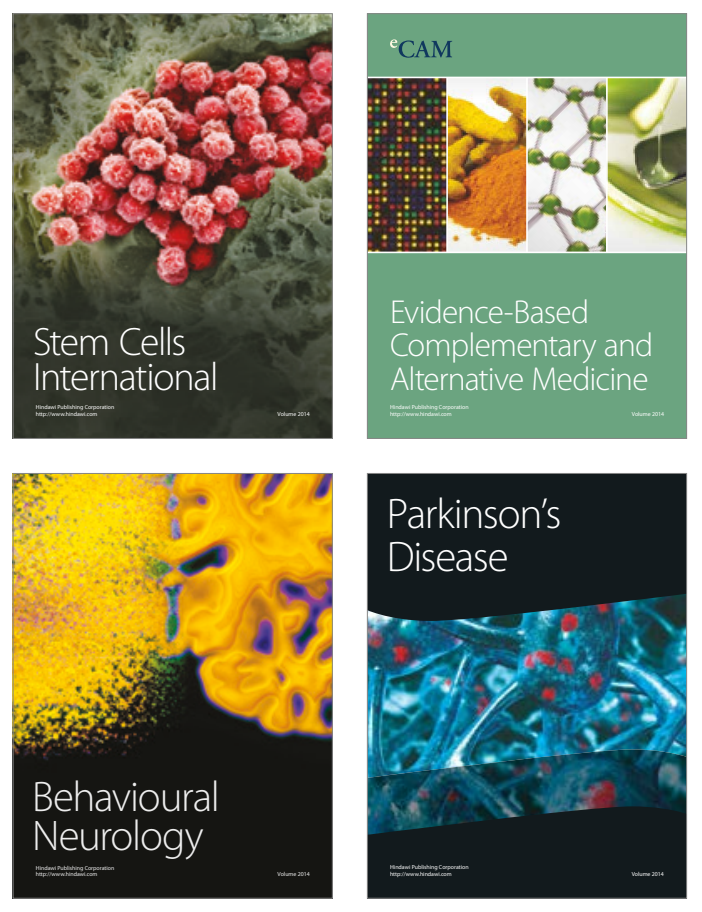
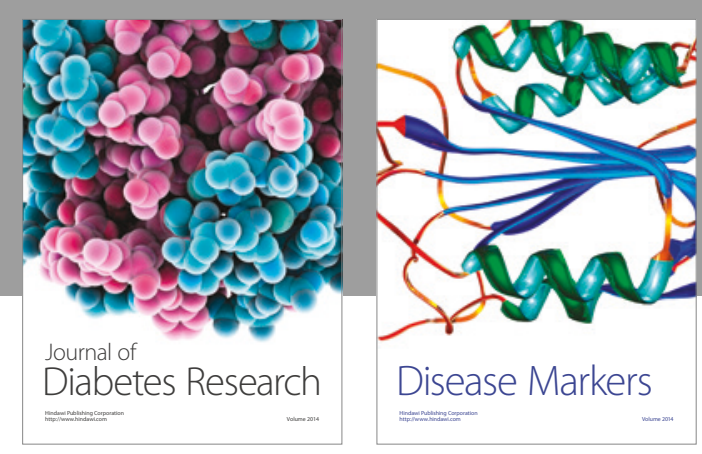

Disease Markers
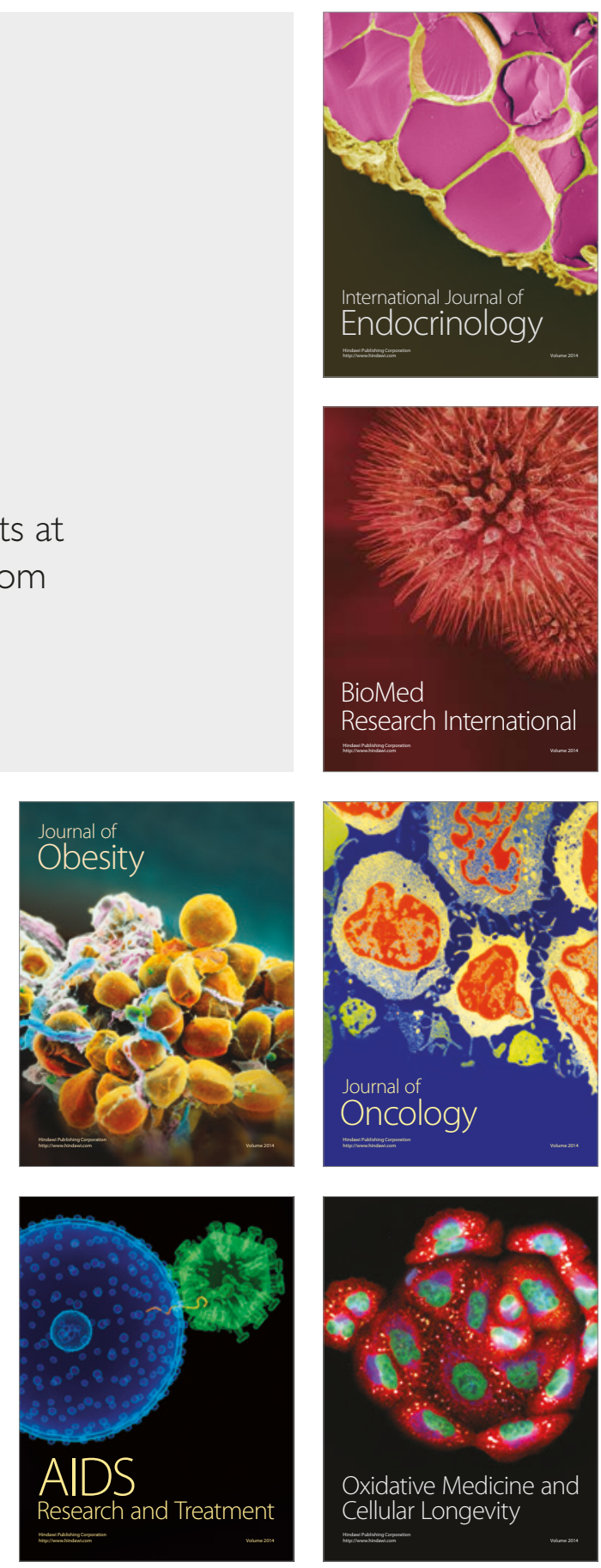